hemodynamics in the contralateral ICA, thus increasing the risk of IA (intracranial aneurysm) formation in the distribution of the non-stenotic artery. The prevalence of IA is higher in patients with ICA stenosis, likely due to alterations in intracranial hemodynamics. We present a patient with aneurysmal subarachnoid hemorrhage who was found to have multiple flowrelated left-sided anterior circulation IA and a chronic right ICA occlusion. We discuss our patient, the considerations for management in this challenging clinical scenario, and review the literature as it pertains to the association of ICA stenosis and IA.

Case presentation A 50-year-old female with a chronic asymptomatic right ICA occlusion presented with diffuse subarachnoid hemorrhage. Emergent angiography revealed left-sided A1-A2 junction, paraclinoid, left middle cerebral artery (MCA) bifurcation, and left anterior temporal artery aneurysms. Brisk filling of the right anterior circulation via the anterior communicating artery (ACOM) was also identified, signifying increased demand on the left ICA circulation.

Treatment/Result Complete obliteration of the A1-A2 junction, paraclinoid, and MCA bifurcation aneurysms was achieved with coil embolization. The anterior temporal artery aneurysm was obliterated with clipping approximately 6 weeks following the hemorrhage. All coiled aneurysms remained obliterated at the time of the 6-week follow-up angiogram. The patient made a complete neurologic recovery and is living independently. Six-month follow-up MRA showed complete occlusion of all treated aneurysms.

Conclusion A review of published case reports/studies of patients with concurrent ICA stenosis and IA revealed a higher number of patients with multiple aneurysms contralateral $(25 \%)$ to, rather than ipsilateral to $(6 \%)$, the ICA stenosis. We present a patient with a chronic asymptomatic right ICA occlusion and aneurysmal $\mathrm{SAH}$, who was found to have multiple, likely flow-related, left-sided anterior circulation aneurysms. All aneurysms were obliterated with a combination of endovascular and microsurgical techniques and the patient made a complete recovery.

Disclosures M. Mathkour: None. E. McCormack: None. C. Werner: None. P. Amenta: None.

\section{E-007 MUTIMODALITY TREATMENT FOR COIL MIGRATION DURING ENDOVASCULAR EMBOLIZATION}

S Chung*, S Chang. Neurosurgery, Eulji University Hospital, Daejeon, Korea, republic of

\subsection{6/neurintsurg-2019-SNIS.82}

Introduction Distal coil migration during endovascular treatment of intracranial aneurysm occurs in $2 \sim 6 \%$ of cases. As endovascular coil embolization of aneurysms has become popular, the incidence of intra-procedural coil migration increases. The consequences of coil migration vary significantly from asymptomatic to as severe as large territory cerebral infarction. However, delayed removal of migrated coil could leave a permanent neurological deficit.

Materials and methods Unintended coil migration occurred in $4(0.81 \%)$ patients among approximately 500 patients treated between Dec 2013 and Jul 2017 in Eulji university hospital. We report three cases of endovascular retrieval performed with snare technique and retrieval stent technique, and one case with microsurgical extraction. Surgical or endovascular removal of migrated coil is commonly used.

Results All 4 patients with coil migration were treated successfully immediately. For 2 cases, we used Amplatz Goose Neck Snare device to remove coil. And Solitare stent was used for retrieval and coil for 1 case was successfully removed. In last case, All endovascular retrieval technique failed and subarachnoid hemorrhage was combined. Due to emergent situation microsurgical craniotomy and arteriotomy was performed and

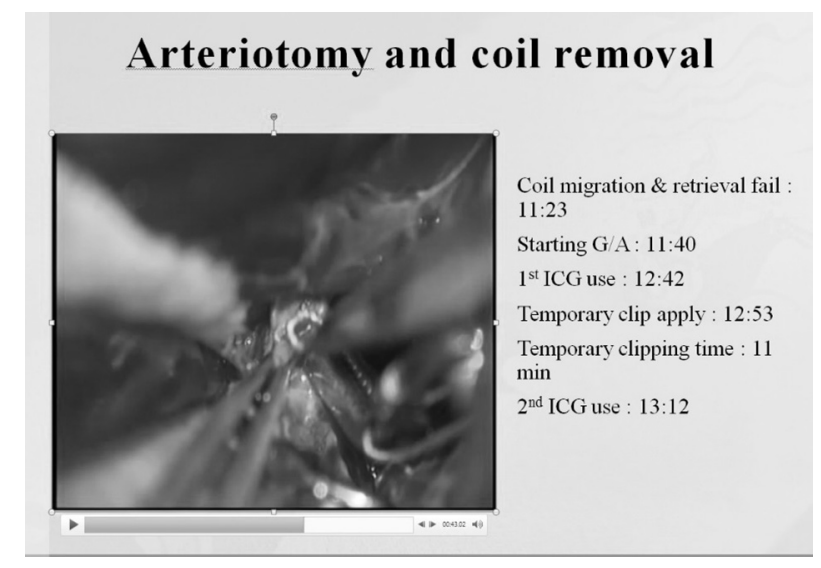

Abstract E-007 Figure 1

\section{Complication \& Salvage procedure}

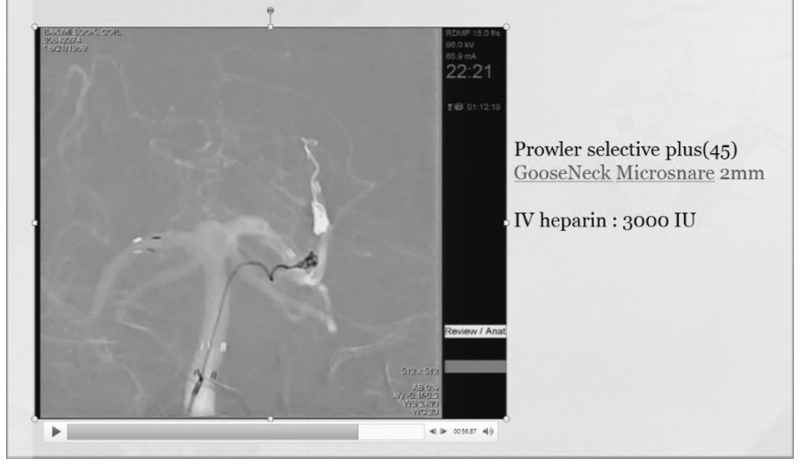

Abstract E-007 Figure 2

migrated coil was removed. All patients recovered without any complication.

Conclusion Migration of coil following embolization of an intracranial aneurysm is rare but can be a fatal complication. Recently the incidences and different results of management have been increasingly reported. In our institution, we performed three cases of endovascular retrieval and one case of surgical retrieval of migrated coil during endovascular coil embolization of intracranial aneurysm. In such urgent situation, operator should consider not only the retrieval of migrated coil but also the consequences following the event such as intracranial hemorrhage or cerebral infarction. Decision between surgical or endovascular retrieval of migrated coil should lead to avoid devastating consequences.

Disclosure S. Chung: None. S. Chang: None. 